\title{
MALDI-TOF MS Assessment to Identify Environmental Mycobacteria
}

\author{
Camilla Pereira de Paula Uzam ${ }^{\#}$, Urze Adomaitis Brianesi ${ }^{\#}$, Camila Romagnoli1, Karen \\ Machado Gomes ${ }^{2}$, Rafael Silva Duarte ${ }^{2}$, Erica Chimara ${ }^{3}$, Julio Cezar Franco de Oliveira1, \\ Marcelo Affonso Vallim', Renata Castiglioni Pascon'1, Cristina Viana-Niero ${ }^{{ }^{*}}$ \\ ${ }^{1}$ Instituto de Ciências Ambientais, Químicas e Farmacêuticas da Universidade Federal de São Paulo, Diadema, \\ Brasil \\ ${ }^{2}$ Instituto de Microbiologia Paulo de Góes da Universidade Federal do Rio de Janeiro, Rio de Janeiro, Brasil \\ ${ }^{3}$ Núcleo de Tuberculose e Micobacterioses do Instituto Adolfo Lutz, São Paulo, Brasil \\ Email: ${ }^{*}$ cristina.viana@unifesp.br
}

Received 17 July 2015; accepted 23 August 2015; published 26 August 2015

Copyright (C) 2015 by authors and Scientific Research Publishing Inc.

This work is licensed under the Creative Commons Attribution International License (CC BY).

http://creativecommons.org/licenses/by/4.0/

(c) (i) Open Access

\begin{abstract}
Over the past few decades, there has been a significant increase in the number of mycobacterial species described. Currently, the genus Mycobacterium consists of 170 species. Most species are called nontuberculous mycobacteria (NTM) and are potentially or rarely pathogenic and ubiquitous. One of the main challenges in mycobacteriology is the rapid and precise identification of these microorganisms. In this work, we compared two protein extraction protocols for the identification of 38 reference strains and clinical isolates, representing 27 species, by mass spectrometry (MALDI-TOF MS) to subsequently use the best method for identifying environmental mycobacteria. The results obtained with reference strains and clinical isolates showed that protocol A was effective in identifying $92.1 \%$ of mycobacterial specimens at the species level and protocol $B, 50 \%$. Therefore, protocol A was evaluated for the rapid identification of 27 environmental mycobacterial isolates. These isolates were subjected to PCR-restriction enzyme analysis (PRA-hsp65). Two isolates were misidentified by PRA-hsp65, whereas MALDI-TOF MS was able to identify them correctly. The results were confirmed by hsp65 and $16 \mathrm{~S}$ rRNA gene sequencing. Mass spectrometry has the advantage of being a simpler and faster technique than PRA-hsp65, and our results showed that MALDI-TOF MS is a valuable tool for the identification of environmental mycobacterial isolates.
\end{abstract}

\section{Keywords}

MALDI-TOF MS, Environmental Mycobacteria, Identification, PRA-Hsp65

\footnotetext{
*Corresponding author.

${ }^{\#}$ Uzam P.P.C. and Brianesi A.U. contributed equally to this work.
} 


\section{Introduction}

Currently, the genus Mycobacterium consists of 170 species and 13 subspecies

(www.bacterio.net/mycobacterium.html). Most species are considered potentially or rarely pathogenic and are called nontuberculous mycobacteria (NTM). This group is widely found in environments shared by humans and animals [1]-[4]. NTM has demonstrated a potential for the degradation of xenobiotic substances [5]-[8]. In the medical field, they have gained interest due to the increased number of infections associated with invasive procedures, such as anesthetic treatments and surgeries, as well as numerous reports of infections associated with pulmonary diseases such as cystic fibrosis [9]-[13]. NTM infections are also characterized by scarce treatment options related to multidrug resistance.

Precise identification at the species level may be useful for the characterization of isolates with biotechnological potential and for directing empiric antimicrobial therapy and choosing relevant drugs for susceptibility tests, according to the American Thoracic Society. Classical identification of mycobacteria is based on phenotypic characteristics and biochemical tests. Besides being time-consuming and laborious, this approach is also limited in identifying all species, and often it has been associated with molecular techniques. PCR-restriction enzyme analysis (PRA) using the hsp65 gene as the target, which encodes the $65-\mathrm{kDa}$ heat shock protein, has been widely used for the identification of mycobacteria [14]-[17]. One limitation of this technique is that different species can share the same restriction profile and that a species may have more than one corresponding profile. The gene encoding 16S rRNA is used for bacterial taxonomy studies, and the high interspecific similarity of the genus Mycobacterium does not allow identification of all mycobacterial species by this method [18]. The joint analysis of different fragments of essential genes (16S rRNA, hsp65, rpoB, sodA) increases the discriminatory power for species identification [18]. These techniques require intensive work, and therefore, the rapid identification of mycobacteria is still a challenge. Alternatively, some studies have proposed the use of matrix-assisted laser desorption/ionization time-of-flight mass spectrometry (MALDI-TOF MS) for the rapid identification of mycobacteria [19]-[23]. Although several protocols for protein extraction have been proposed, there is no consensus on the best protocol to be used for the identification of mycobacteria. Furthermore, the effect of microorganism inactivation by heat has been evaluated, and it has been shown that the quality of the spectrum decreases with increasing temperature [24]. It was also recently reported that the conditions of microorganism cultivation also influence the spectrum [25] [26]. However, in another study, the effect of culture age, chilling or freezing the lysates and freeze-thaw cycles did not have any impact on spectrum quality [27]. Furthermore, its use of MALDI-TOF MS for environmental isolates remains to be further explored, since only one reported study included two environmental isolates in their analysis [28].

In this paper, we compared two protein extraction protocols for preparing samples for MALDI-TOF MS analysis (protocols A and B) using reference strains and clinical isolates. Furthermore, we evaluated the application of MALDI-TOF MS for the identification of environmental mycobacteria and compared it with the results obtained with PRA-hsp65.

\section{Material and Methods}

\subsection{Bacterial Strains}

Twenty reference strains from Instituto Adolfo Lutz (IAL Collection) and Universidade Federal do Rio de Janeiro (UFRJ Collection) and also 18 clinical isolates previously identified by PRA-hsp65 at IAL were used in the present study (Table 1). These strains were chosen because the species are represented in the Bruker Daltonics database and also because of their availability in our laboratory. Additionally, 27 environmental mycobacterial isolates obtained in the period of November 2011 to May 2012 from four aquatic environments of São Paulo Zoo Park Foundation (FPZSP) were included in this study: sewage treatment plant, Lake 70 and two springs, called 1 and 2 (Figure 2, unpublished data). These isolates were deposited in the microbial collection of FPZSP. Mycobacteria were grown on Middlebrook7H10-OADC (oleic acid-albumin-dextrose-catalase) at $30^{\circ} \mathrm{C}$ or $37^{\circ} \mathrm{C}$, depending on the optimum growth temperature of each species.

\subsection{Matrix-Assisted Laser Desorption/Ionization Time-of-Flight Mass Spectrometry (MALDI-TOF-MS) and Analysis}

All 65 mycobacterial isolates were analyzed by MALDI-TOF MS. The reference strains and clinical isolates 
Table 1. Reference strains and clinical isolates used in this study for comparative analysis between two protein extraction protocols for MALDI-TOF-MS.

\begin{tabular}{|c|c|}
\hline Mycobacterium species & Strain \\
\hline M. abscessus 1 & ${ }^{\mathrm{a}}$ IAL 1554 \\
\hline M. abscessus 1 & ${ }^{\mathrm{a}}$ IAL 1616 \\
\hline M. abscessus 1 & a IAL 1669 \\
\hline M. abscessus & bैATCC 19,977 \\
\hline M. asiaticum 1 & ${ }^{\mathrm{a}}$ IAL 184 \\
\hline M. asiaticum & ${ }^{\mathrm{b}}$ ATCC 25,272 \\
\hline M. aurum & ${ }^{\mathrm{b}} \mathrm{ATCC} 23,366$ \\
\hline M. austroafricanum & ${ }^{\mathrm{b}}$ ATCC 33,464 \\
\hline M. avium 1 & ${ }^{\mathrm{a}}$ IAL 1550 \\
\hline M. brisbanense 1 & ${ }^{\mathrm{a}}$ IAL 935 \\
\hline M. chelonae 1 & ${ }^{\mathrm{a}}$ IAL 1717 \\
\hline M. chelonae & ${ }^{\mathrm{b}}$ ATCC 35,752 \\
\hline M. cosmeticum 1 & ${ }^{\mathrm{a}}$ IAL 954 \\
\hline M. diernhoferi & ${ }^{\mathrm{b}}$ ATCC 19,340 \\
\hline M. fortuitum 1 & ${ }^{\mathrm{a}}$ IAL 727 \\
\hline M. fortuitum 1 & ${ }^{\mathrm{a}}$ IAL 970 \\
\hline M. fortuitum & ${ }^{\mathrm{b}}$ ATCC 6841 \\
\hline M. gordonae 3 & aAL 1523 \\
\hline M. gordonae 3 & ${ }^{\mathrm{a}}$ IAL 1732 \\
\hline M. gordonae 3 & ${ }^{\mathrm{a}}$ IAL 862 \\
\hline M. intracellulare & ${ }^{\mathrm{b}}$ ATCC 13,950 \\
\hline M. kansasii & ${ }^{\mathrm{b}} \mathrm{INCQS} 07002$ \\
\hline M. mucogenicum 1 & ${ }^{\mathrm{a}}$ IAL 1648 \\
\hline M. nonchromogenicum & ${ }^{\mathrm{b}}$ ATCC 19,530 \\
\hline M. parafortuitum & ${ }^{\mathrm{b}}$ ATCC 19,686 \\
\hline M. peregrinum 1 & ${ }^{\mathrm{a}}$ IAL 1319 \\
\hline M. peregrinum 1 & ${ }^{\mathrm{a}}$ IAL 196 \\
\hline M. phlei & ${ }^{\mathrm{b}}$ ATCC 11,758 \\
\hline M. porcinum & ${ }^{\mathrm{b}}$ ATCC 33776 \\
\hline M. pulveris & ${ }^{\mathrm{b}}$ ATCC 35154 \\
\hline M. rhodesiae & ${ }^{\mathrm{b}}$ ATCC 27024 \\
\hline M. scrofulaceum 1 & ${ }^{\mathrm{a}}$ IAL 1750 \\
\hline M. scrofulaceum & ${ }^{\mathrm{b}}$ ATCC 19,981 \\
\hline M. smegmatis & ${ }^{\mathrm{b}}$ ATCC 14,468 \\
\hline M. szulgai & ${ }^{\mathrm{b}}$ ATCC 35,799 \\
\hline M. terrae 2 & ${ }^{\mathrm{a}}$ IAL 1684 \\
\hline M. thermoresistibile & ${ }^{\mathrm{b}}$ ATCC 19,527 \\
\hline M. vaccae & ${ }^{\mathrm{b}}$ ATCC 15,483 \\
\hline
\end{tabular}

${ }^{\mathrm{a}}$ Clinical isolates previously identified by PRA-hsp65; ${ }^{\mathrm{b}}$ Reference collection isolate. 
were used for comparative analysis between the two protein extraction protocols, one described by El Khéchine et al. (2011) and the other by Balázová et al. (2014), recommended by Bruker Daltonics (inactivated mycobacteria bead preparation method inMbpm) and designated A and B, respectively. After protein extraction, $1 \mu \mathrm{L}$ of the supernatant was placed on a Micro Scout Plate (MSP) 96 polished steel target plate (Bruker Daltonics GmbH, Germany) in triplicate for each sample. The samples were allowed to dry at room temperature. Each sample was overlaid with $1 \mu \mathrm{L}$ of a saturated solution of a-cyano-4-hydroxy-cinnamic acid (Sigma, USA) in 50\% acetonitrile-2.5\% trifluoroacetic (Sigma, USA) and the samples were air-dried before being processed in the mass spectrometer. Bacterial Test Standard (Escherichia coli protein extract; Bruker Daltonics) was used for equipment calibration according to Bruker Daltonics. The analysis was performed using a Microflex LT mass spectrometer (Bruker Daltonics) at $337 \mathrm{~nm}$ with the FlexControl software (version 3.0, Bruker Daltonics). Positive linear mode was used to record spectra (laser frequency, $40 \mathrm{HzA}$; ion source 1 voltage, $20 \mathrm{kV}$; ion source 2 voltage, $18.6 \mathrm{kV}$; lens voltage, $7.5 \mathrm{kV}$; mass range, 2000 to 20,000 Da). For each spectrum, 240 shots in 50-shot steps from different positions of the target spot (automatic mode) were collected. Each spectrum was compared with the Bruker Daltonics database (Mycobacteria Library 1.0) using the BioTyper software (version 3.0, Bruker Daltonics). Outcomes of the pattern-matching process were expressed with identification score value from 0 to 3. According to the software manufacturer (Bruker Daltonics), scores were interpreted as follows: $<1.700$, unreliable identification; 1.7 to 1.999, probable genus identification; 2.000 to 2.299, probable species identification; and equal to or greater than 2.300 , reliable species identification. The spectra obtained for each sample were transformed to "main spectra" (MSPs) for further analysis of similarity by BioNumerics 7.5 (Applied Maths, Sint Martens Latem, Belgium). The similarity matrix between spectra was inferred on the basis of the MSPs using Pearson's method, with a positional tolerance of $2 \%$, and a dendrogram was then constructed using UPGMA.

\subsection{PCR Restriction Enzyme Analysis (PRA-hsp65)}

Nucleic acids were extracted by lysing bacterial cells. Briefly, colonies were transferred to a $1.5 \mathrm{~mL}$ microcentrifuge tube and incubated in $10 \mathrm{mMTris}-1 \mathrm{mM}$ EDTA for $20 \mathrm{~min}$ at $80^{\circ} \mathrm{C}$ to inactivate the mycobacteria. The sample was centrifuged at 10,000 xg and $5 \mu \mathrm{L}$ of the thermolysate was used for PCR. Twenty-seven environmental mycobacterial isolates were analyzed by PRA-hsp65 [29]. Briefly, the 441-bp fragment of the $h s p 65$ gene was amplified with primers TB11 (5' ACCAACGATGGTGTGTCCAT) and TB12 (5' CTTGTCGAACCGC ATACCCT). Amplicons were digested separately with BstEII (Promega) and HaeIII (Invitrogen). Digestion products were visualized after electrophoresis in agarose gels, and digestion fragment size was estimated by visual analysis and using the BioNumerics v. 7.5 program (Applied Maths). The digestion patterns obtained were compared to the PRASITE internet database (http://app.chuv.ch/prasite/index.html).

\subsection{Sequencing of $h s p 65$ and 16 S rRNA}

Isolates with discordant results between PRA-hsp65 and MALDI-TOF-MS and isolates grouped by PRA-hsp65 and MALDI-TOF-MS profiles but not identified were analyzed by sequencing of $h s p 65$ and 16S rRNA genes. Five microliters of the thermolysate were used for amplification of hsp65 and 16S rRNA genes by PCR. A 667-bp fragment of $h s p 65$ gene was amplified with primers hsp667-forward (5' GGCCAAGACAATTGCGTACG) and hsp667-reverse (5' GGAGCTGACCAGCAGGATG) [30], it was regarded as the internal fragment of 401 bp corresponding to the region analyzed by PRA-hsp65 without the sequences of the primers. Complete sequences of the 16S rRNA gene (1483 - 1489 bp) were amplified using primer pair fD1-rP2 [31]. The sequencing reactions were performed with the same primers used for amplification of the $h s p 65$ and 16S RNA genes. As for the latter, six internal primers were used as described by Adekambi and Drancourt (2004). Products of sequencing reactions were recorded with an ABI Prism 3100 DNA sequence following the manufacturer's standard protocol (Perkin Elmer Applied Biosystems). The consensus sequences generated by BioNumerics v7.5 (trimmed from at both ends) and were identified by similarity analysis with the sequences in the GenBank data-

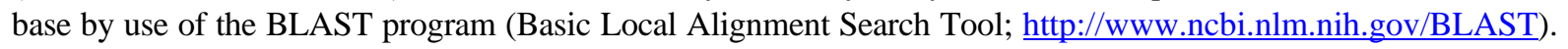
The cutoff used for analysis of 16S rRNA and $h s p 65$ genes for identification was equal to or greater than 99 and 97\% identity, respectively [31] [32]. The consensus sequences obtained in this study were deposited in GenBank under accession numbers KP76838 and, KR779818 (hsp65) and KP768388 and KR779819 (16S rRNA). 


\section{Results}

The first goal of this study was to compare two protocols of protein extraction for the identification of mycobacteria by mass spectrometry (MALDI-TOF) to subsequently use the best method for identifying environmental isolates. To achieve these aims, 38 clinical and reference samples of mycobacteria representing 27 species were chosen. Protocol A identified 35 (92.1\%) isolates at the species level and three (7.9\%) at the genus level, while protocol B identified 19 (50\%) isolates at the species level, 18 (47.3\%) at the genus level and one isolate (2.6\%) could not be identified because it showed a score below 1.700, when compared to the database (Figure 1(a)). Protocol A generated the highest number of detectable peaks when compared to protocol B for all samples. A comparative analysis of the spectra obtained by protocols A and B and the spectra deposited in the database revealed greater similarity between the spectra obtained with protocol A and spectra in the database (Figure 1(b)). After comparing the protein extraction protocols, the collection of 27 environmental isolates was analyzed by protocol A. Of the 27 environmental isolates tested, 12 (44.4\%) were identified at the species level; although the other 15 showed good-quality spectra, they could not be reliably identified using the Bruker Daltonics database (no score values, Figure 2).

All environmental isolates were also analyzed by PRA-hsp65. Seventeen isolates (62.9\%) were identified at the species level by PRA-hsp65: M. alvei 1, M. aubagnense 1, M. chelonae 1, M. nebraskense 1 , M. neoaurum 1, M. parafortuitum 1 and $M$. peregrinum2/porcinum1/septicum1. Ten isolates were separated into four groups according to the restriction profile generated by BstEII and HaeIII restriction enzymes. However their profiles were not found in the PRASITE database, and therefore, we designated them as "new” (Figure 2). When comparing the two methods, we noted that the isolates identified by PRA-hsp65 as M. nebraskense 1 and M. aubagnense 1 , were not identified by MALDI-TOF MS because matching spectrum was not represented within the Bruker Daltonics Database. Three other isolates (MYC 78, 79 and 101) not identified by PRA-hsp65 or Maldi-TOF MS were subjected to sequencing of hsp65 and 16S rRNA. Sequences from these isolates showed the same sequences for these genes, and only the sequences from isolate MYC78 were deposited in the GenBank database. The hsp65 gene sequence was $96.7 \%$ identical to that of $M$. longobardum DSM45394 (access number JN571199.1), and the 16S rRNA sequence showed 98.7\% identity with $M$. senuense strain 05-832 (access number NR043905.1), it was not possible to complete the identification.

The identification of two isolates (MYC100 and 106) were in disagreement when considering PRA-hsp65 and MALDI-TOF MS techniques. The identification by PRA- $h s p 65$ generated a profile shared by three species, $M$. peregrinum2/porcinum1/septicum1, and MALDI-TOF MS identified both isolates as M. brisbanense. The sequencing analysisof the hsp65 gene fragment from these two isolates showed sequences identical to each other, with 99.5\% identity with $M$. brisbanense DSM 44,680 sequence (access number JF491333.1), thus confirming the identification obtained with MALDI-TOF MSand suggesting a new PRA-hsp65 profile for M. brisbanense. The divergence between PRA-hsp65 and hsp65 gene sequence results was due to a mismatch of two nucleotides at positions 307 (CT) and 310 (TC) of the 401 bp fragment from the hsp65 gene. The changes in these nucleotides resulted in loss of the recognition site for the restriction enzyme BstEII, which is used in PRA-hsp65, and generated a restriction profile compatible with that of other species ( $M$. peregrinum2/septicum1/porcinum1) when analyzed by PRASITE (http://app.chuv.ch/prasite/index.html), erroneously identifying these isolates. The 16S rRNA gene sequences also revealed that the MYC100 and 106 isolates were identical to each other and showed 99.2\% identity with the sequence of M. brisbanense, strain W6743 and access number NR029037.1, thus confirming the identification obtained with MALDI-TOF MS. The sequences of isolate MYC100 were deposited in the GenBank database.

\section{Discussion}

The present study compared two protein extraction protocols for MALDI-TOF MS biotyping using reference strains and clinical isolates, representing 27 mycobacteria species. We chose to test the protocols described by El Khéchine (protocol A) and Bruker Daltonics (protocol B). The choice of protocol A was based on the assumption that the mechanical disruption of the mycobacterial wall step, when carried out in a controlled manner by equipment, it could be a determining factor in obtaining quality spectra for analysis. The reason for protocol B was library availability for data comparison and inclusion of new spectra, expanding the database.

The results showed that protocol A was more successful than protocol B in identifying mycobacterial species by MALDI-TOF MS. The comparative analysis between spectra obtained with the two protocols and the spectra 


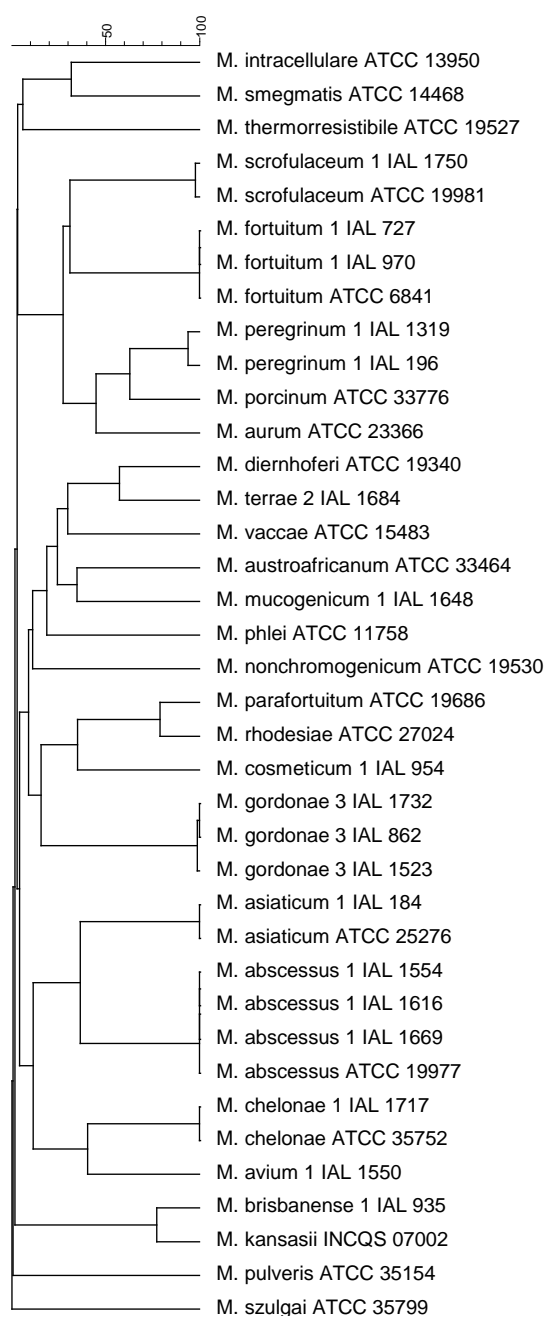

\begin{tabular}{ll}
$>2,300$ & $>2,300$ \\
$>2,300$ & $>2,300$ \\
$>2,300$ & $>2,300$ \\
$>2,300$ & $>2,300$ \\
$>2,300$ & $>2,300$ \\
2,017 & 1,849 \\
2,086 & 1,849 \\
2,031 & 2,003 \\
$>2,300$ & $>2,300$ \\
$>2,300$ & $>2,300$ \\
$>2,300$ & 1,732 \\
$>2,300$ & $>2,300$ \\
$>2,300$ & 2,218 \\
$>2,300$ & $>2,300$ \\
$>2,300$ & $>2,300$ \\
$>2,300$ & $<1,700$ \\
$>2,300$ & $>2,300$ \\
$>2,300$ & $>2,300$ \\
$>2,300$ & $>2,300$ \\
$>2,300$ & 2,150 \\
$>2,300$ & 2,167 \\
$>2,300$ & $>2,300$ \\
$>2,300$ & 1,963 \\
$>2,300$ & 1,959 \\
$>2,300$ & 1,878 \\
$>2,300$ & $>2,300$ \\
$>2,300$ & 2,252 \\
$>2,300$ & 2,169 \\
$>2,300$ & 2,232 \\
$>2,300$ & 2,245 \\
$>2,300$ & 2,140 \\
$>2,300$ & $>2,300$ \\
$>2,300$ & $>2,300$ \\
$>2,300$ & 1,800 \\
$>2,300$ & $>2,300$ \\
$>2,300$ & 1,997 \\
& $>2,300$ \\
\hline 2,300 \\
\hline 200
\end{tabular}

(a)

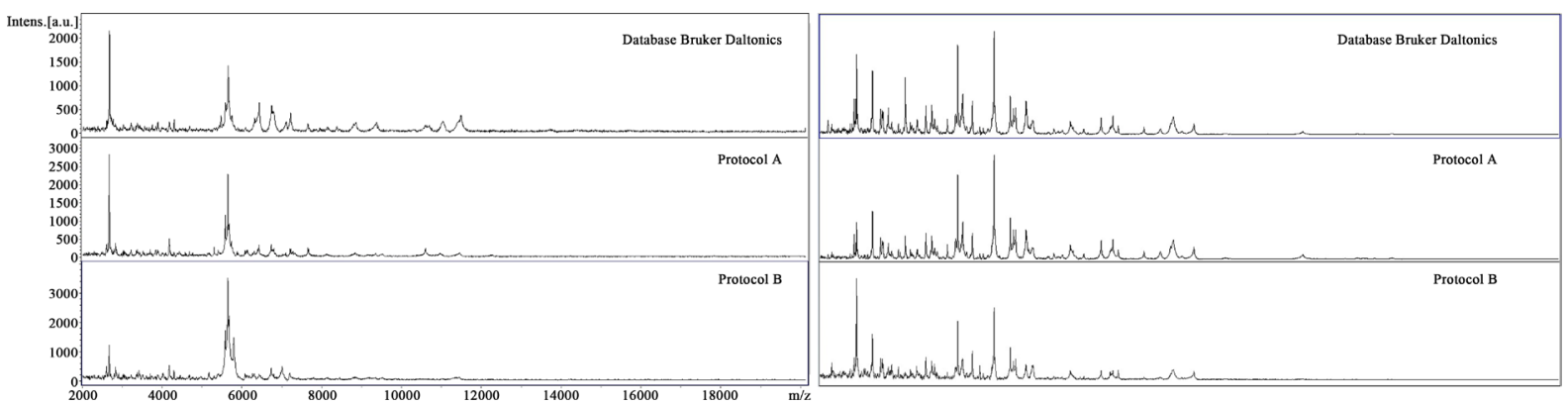

(b)

Figure 1. Identification of reference strains and clinical isolates by MALDI-TOF-MS. (a) Dendrogram of protein mass profiles grouped by Pearson coefficient with $2 \%$ tolerance, using the BioNumerics program v. 7.5 , and score obtained with protocols A and B. (b) Comparative spectral fingerprint of M. abscessus IAL1669 (left) and M. smegmatis ATCC14468 (right) obtained with Microflex LT mass spectrometer (Bruker Daltonics). m/z, mass-to-charge ratio.

deposited in the Brucker Daltonics database revealed that the spectra obtained with protocol A were more similar to those deposited in the database. Both protocols A and B used heat to inactivate the microorganisms at $95^{\circ} \mathrm{C}$ for $1 \mathrm{~h}$ and $30 \mathrm{~min}$, respectively. They also used beads for mechanical disruption of the mycobacterial wall. In protocol A, disruption of the mycobacterial wall took place by controlled shaking in a Fastprep instrument, whereas vortexing was used in protocol B. Shitikov and colleagues observed lower spectrum quality with great- 


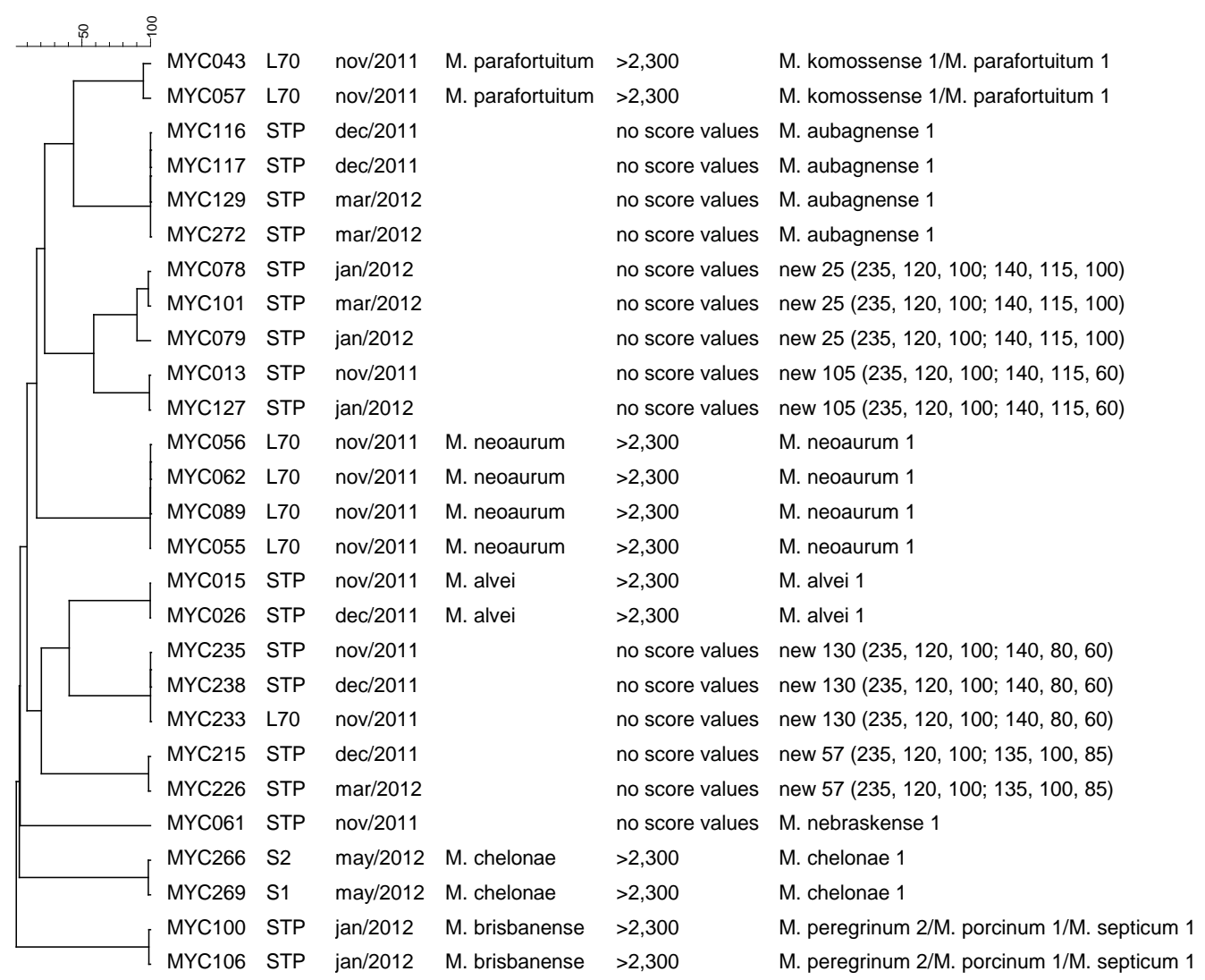

Figure 2. Identification of environmental mycobacterial isolates by MALDI-TOF MS and PRA-hsp65. Dendrogram of protein mass profiles generated using bionumerics program v. 7.5. (Applied maths, belgium). L70 = Lake 70, STP = sewage treatment plant, $\mathrm{S} 1$ = Spring $1, \mathrm{~S} 2$ = Spring 2 .

er sample heating [24]. In protocol A, the samples were subjected to heating twice as long as in protocol, but this observation was not confirmed by the samples studied. Our hypothesis was that the most important factor in obtaining quality spectra is the efficiency of disrupting the mycobacterial wall, which in protocol A, occurred through controlled mechanical agitation using equipment [23]. It was shown in a previous study that changes in the extraction protocol generated differences in the spectra [23]. Another work evaluated the effect of cultivation time, cooling of the lysate and freezing cycles on the quality of the spectrum and found no adverse impact on spectrum quality or identification [27]. These findings combined with our results suggest that the disruption of the mycobacterial wall is a critical step for efficient protein extraction, quality spectrum and consequently identification. Still, our results suggest that samples prepared with protocol A, though different from the protocol used to construct the Bruker Daltonics database, are amenable to analysis, and moreover, spectrum quality obtained with protocol A was comparable to that deposited in the Bruker Daltonics database. However, other authors tested the same protocols (A and B) and concluded that they were not amenable to analysis in the Bruker Daltonics database [25]. We believe that the differences may be related to the mycobacterial cell wall break down process. While we used the Fastprep at full speed for $3 \mathrm{~min}$ as described in the original protocol [23], Balazova and collaborators used a thermomixer at $1400 \mathrm{rpm}$ for $2 \mathrm{~min}$. The comparison between the resulting spectra generated by protocols A and B using reference and clinical strains led us to choose protocol A for the assessment of 27 unidentified environmental mycobacterial isolates by mass spectrometry. Concomitantly, the environmental isolates were analyzed by PRA-hsp65.

Mass spectrometry identified, at the species level, $44.4 \%$ of the environmental isolates while the PRA-hsp65 technique identified $62.9 \%$. This difference occurred because six isolates identified as $M$. nebraskense and $M$. aubagnense by PRA-hsp65 were not identified by MALDI-TOF MS, since the database used for analysis does not have a spectrum for these microorganisms in its records. These findings strongly suggest that it is important to broaden the spectrum database. Most species of environmental mycobacteria identified in this study have 
been described as being responsible for infections in humans and animals [12] [16] [33]-[37]. Ten environmental isolates (37\%) could not be identified by any of these techniques. These results corroborate studies reporting that $48 \%$ to $61 \%$ of environmental mycobacteria isolated from water cannot be identified by phenotypic and molecular methods, demonstrating the importance of evaluating new identification techniques [1] [14] [38]. The number of new species of mycobacteria has been increasing exponentially making identification a challenge [39].

The joint analysis of the results obtained with MALDI-TOF and PRA-hsp65 revealed a disagreement in two isolates identified as $M$. brisbanense and M. peregrinum2/porcinum1/septicum1, respectively. The sequencing of the hsp65 gene fragment showed two nucleotide changes resulting in the loss of recognition site for the restriction enzyme BstEII, which was used in the PRA-hsp65 method, erroneously identifying these isolates. The sequencing of the 16S rRNA gene showed that these two isolates shared $99.2 \%$ of identity to $M$. brisbanense sequence thus confirming the identification obtained with MALDI-TOF MS.

In conclusion, our results showed that MALDI-TOF MS and PRA-hsp65 had the potential for the identification of environmental isolates, considering an available database with profiles for comparison. Mass spectrometry has the advantage of being a simpler and faster technique than PRA-hsp65.

In work we were able to test a fast and efficient method for protein extraction followed by mass spectrometry biotyping of environmental mycobacterial isolates, as an answer to the growing need for a rapid and suitable method for identification of the increasing number of new mycobacterial species to be characterized.

\section{Acknowledgments}

The authors would like to acknowledge Dr. Diego Assis for his assistance with MALDI-TOF MS and the staff from São Paulo Zoo Park Foundation for technical help. This work was supported by Fundação de Amparo à Pesquisa doEstado de São Paulo (FAPESP; process No. 2010/52641-1, 2011/13140-0, 2011/13271-7). Dr. A. Leyva helped with English editing of the manuscript.

\section{References}

[1] Le Dantec, C., Duguet, J.P., Montiel, A., Dumoutier, N., Dubrou, S. and Vincent, V. (2002) Occurrence of Mycobacteria in Water Treatment Lines and in Water Distribution Systems. Applied and Environmental Microbiology, 68, 5318-5325. http://dx.doi.org/10.1128/AEM.68.11.5318-5325.2002

[2] Parashar, D., Chauhan, D.S., Sharma, V.D., Chauhan, A., Chauhan, S.V. and Katoch, V.M. (2004) Optimization of Procedures for Isolation of Mycobacteria from Soil and Water Samples Obtained in Northern India. Applied and Environmental Microbiology, 70, 3751-3753. http://dx.doi.org/10.1128/AEM.70.6.3751-3753.2004

[3] Radomski, N., Cambau, E., Moulin, L., Haenn, S., Moilleron, R. and Lucas, F.S. (2010) Comparison of Culture Methods for Isolation of Nontuberculous Mycobacteria from Surface Waters. Applied and Environmental Microbiology, 76, 3514-3520. http://dx.doi.org/10.1128/AEM.02659-09

[4] Kankya, C., Muwonge, A., Djonne, B., Munyeme, M., Opuda-Asibo, J., Skjerve, E., Oloya, J., Edvardsen, V. and Johansen, T.B. (2011) Isolation of Non-Tuberculous Mycobacteria from Pastoral Ecosystems of Uganda: Public Health Significance. BMC Public Health, 11, 320. http://dx.doi.org/10.1186/1471-2458-11-320

[5] Heitkamp, M.A., Freeman, J.P., Miller, D.W. and Cerniglia, C.E. (1988) Pyrene Degradation by a Mycobacterium sp.: Identification of Ring Oxidation and Ring Fission Products. Applied and Environmental Microbiology, 54, 2556-2565.

[6] Miller, C.D., Hall, K., Liang, Y.N., Nieman, K., Sorensen, D., Issa, B., Anderson, A.J. and Sims, R.C. (2004) Isolation and Characterization of Polycyclic Aromatic Hydrocarbon-Degrading Mycobacterium Isolates from Soil. Microbial ecology, 48, 230-238. http://dx.doi.org/10.1007/s00248-003-1044-5

[7] Maciel, H., Mathis, H., Lopes Ferreira, N., Lyew, D., Guiot, S., Monot, F., Greer, C.W. and Fayolle-Guichard, F. (2008) Use of Mycobacterium austroafricanum IFP 2012 in a MTBE-Degrading Bioreactor. Journal of Molecular Microbiology and Biotechnology, 15, 190-198.

[8] Hennessee, C.T., Seo, J.S., Alvarez, A.M. and Li, Q.X. (2009) Polycyclic Aromatic Hydrocarbon-Degrading Species Isolated from Hawaiian Soils: Mycobacterium crocinum sp. nov., Mycobacterium pallens sp. nov., Mycobacterium rutilum sp. nov., Mycobacterium rufum sp. nov. and Mycobacterium aromaticivorans sp. nov. International Journal of Systematic and Evolutionary Microbiology, 59, 378-387. http://dx.doi.org/10.1099/ijs.0.65827-0

[9] Hofling-Lima, A.L., de Freitas, D., Sampaio, J.L., Leao, S.C. and Contarini, P. (2005) In Vitro Activity of Fluoroquinolones against Mycobacterium abscessus and Mycobacterium chelonae Causing Infectious Keratitis after LASIK in Brazil. Cornea, 24, 730-734. http://dx.doi.org/10.1097/01.ico.0000154411.07315.0a 
[10] Viana-Niero, C., Lima, K.V., Lopes, M.L., Rabello, M.C., Marsola, L.R., Brilhante, V.C., Durham, A.M. and Leao, S.C. (2008) Molecular Characterization of Mycobacterium massiliense and Mycobacterium bolletii in Isolates Collected from Outbreaks of Infections after Laparoscopic Surgeries and Cosmetic Procedures. Journal of Clinical Microbiology, 46, 850-855. http://dx.doi.org/10.1128/JCM.02052-07

[11] Duarte, R.S., Lourenco, M.C., Fonseca Lde, S., Leao, S.C., Amorim Ede, L., Rocha, I.L., Coelho, F.S., Viana-Niero, C., Gomes, K.M., da Silva, M.G., et al. (2009) Epidemic of Postsurgical Infections Caused by Mycobacterium massiliense. Journal of Clinical Microbiology, 47, 2149-2155. http://dx.doi.org/10.1128/JCM.00027-09

[12] Strabelli, T.M., Siciliano, R.F., Castelli, J.B., Demarchi, L.M., Leao, S.C., Viana-Niero, C., Miyashiro, K., Sampaio, R.O., Grinberg, M. and Uip, D.E. (2010) Mycobacterium chelonae Valve Endocarditis Resulting from Contaminated Biological Prostheses. The Journal of Infection, 60, 467-473. http://dx.doi.org/10.1016/j.jinf.2010.03.008

[13] Candido, P.H., Nunes Lde, S., Marques, E.A., Folescu, T.W., Coelho, F.S., de Moura, V.C., da Silva, M.G., Gomes, K.M., Lourenco, M.C., Aguiar, F.S., et al. (2014) Multidrug-Resistant Nontuberculous Mycobacteria Isolated from Cystic Fibrosis Patients. Journal of Clinical Microbiology, 52, 2990-2997. http://dx.doi.org/10.1128/JCM.00549-14

[14] Bland, C.S., Ireland, J.M., Lozano, E., Alvarez, M.E. and Primm, T.P. (2005) Mycobacterial Ecology of the Rio Grande. Applied and Environmental Microbiology, 71, 5719-5727. http://dx.doi.org/10.1128/AEM.71.10.5719-5727.2005

[15] Leao, S.C., Bernardelli, A., Cataldi, A., Zumarraga, M., Robledo, J., Realpe, T., Mejia, G.I., da Silva Telles, M.A., Chimara, E., Velazco, M., et al. (2005) Multicenter Evaluation of Mycobacteria Identification by PCR Restriction Enzyme Analysis in Laboratories from Latin America and the Caribbean. Journal of Microbiological Methods, 61, 193199. http://dx.doi.org/10.1016/j.mimet.2004.11.015

[16] Lee, C.H., You, H.L., Wang, J.W., Tang, Y.F. and Liu, J.W. (2011) Prosthetic Joint Infection Caused by Mycobacterium alvei in an Elderly Patient. Journal of Clinical Microbiology, 49, 3096-3098. http://dx.doi.org/10.1128/JCM.00603-11

[17] Chimara, E., Ferrazoli, L., Ueky, S.Y., Martins, M.C., Durham, A.M., Arbeit, R.D. and Leao, S.C. (2008) Reliable Identification of Mycobacterial Species by PCR-Restriction Enzyme Analysis (PRA)-hsp65 in a Reference Laboratory and Elaboration of a Sequence-Based Extended Algorithm of PRA-hsp65 Patterns. BMC Microbiology, 8, 48. http://dx.doi.org/10.1186/1471-2180-8-48

[18] Devulder, G., de Montclos, M.P. and Flandrois, J.P. (2005) A Multigene Approach to Phylogenetic Analysis Using the Genus Mycobacterium as a Model. International Journal of Systematic and Evolutionary Microbiology, 55, $293-302$. http://dx.doi.org/10.1099/ijs.0.63222-0

[19] Hettick, J.M., Kashon, M.L., Simpson, J.P., Siegel, P.D., Mazurek, G.H. and Weissman, D.N. (2004) Proteomic Profiling of Intact Mycobacteria by Matrix-Assisted Laser Desorption/Ionization Time-of-Flight Mass Spectrometry. Analytical Chemistry, 76, 5769-5776. http://dx.doi.org/10.1021/ac049410m

[20] Pignone, M., Greth, K.M., Cooper, J., Emerson, D. and Tang, J. (2006) Identification of Mycobacteria by MatrixAssisted Laser Desorption Ionization-Time-of-Flight Mass Spectrometry. Journal of Clinical Microbiology, 44, 19631970. http://dx.doi.org/10.1128/JCM.01959-05

[21] Lotz, A., Ferroni, A., Beretti, J.L., Dauphin, B., Carbonnelle, E., Guet-Revillet, H., Veziris, N., Heym, B., Jarlier, V., Gaillard, J.L., et al. (2010) Rapid Identification of Mycobacterial Whole Cells in Solid and Liquid Culture Media by Matrix-Assisted Laser Desorption Ionization-Time of Flight Mass Spectrometry. Journal of Clinical Microbiology, 48, 4481-4486. http://dx.doi.org/10.1128/JCM.01397-10

[22] Saleeb, P.G., Drake, S.K., Murray, P.R. and Zelazny, A.M. (2011) Identification of Mycobacteria in Solid-Culture Media by Matrix-Assisted Laser Desorption Ionization-Time of Flight Mass Spectrometry. Journal of Clinical Microbiology, 49, 1790-1794. http://dx.doi.org/10.1128/JCM.02135-10

[23] El Khechine, A., Couderc, C., Flaudrops, C., Raoult, D. and Drancourt, M. (2011) Matrix-Assisted Laser Desorption/Ionization Time-of-Flight Mass Spectrometry Identification of Mycobacteria in Routine Clinical Practice. PloS ONE, 6, e24720. http://dx.doi.org/10.1371/journal.pone.0024720

[24] Shitikov, E., Ilina, E., Chernousova, L., Borovskaya, A., Rukin, I., Afanas’ev, M., Smirnova, T., Vorobyeva, A., Larionova, E., Andreevskaya, S., et al. (2012) Mass Spectrometry Based Methods for the Discrimination and Typing of Mycobacteria. Infection, Genetics and Evolution: Journal of Molecular Epidemiology and Evolutionary Genetics in Infectious Diseases, 12, 838-845.

[25] Balazova, T., Makovcova, J., Sedo, O., Slany, M., Faldyna, M. and Zdrahal, Z. (2014) The Influence of Culture Conditions on the Identification of Mycobacterium Species by MALDI-TOF MS Profiling. FEMS Microbiology Letters, 353, 77-84. http://dx.doi.org/10.1111/1574-6968.12408

[26] Mather, C.A., Rivera, S.F. and Butler-Wu, S.M. (2014) Comparison of the Bruker Biotyper and Vitek MS Matrix-Assisted Laser Desorption Ionization-Time of Flight Mass Spectrometry Systems for Identification of Mycobacteria Using Simplified Protein Extraction Protocols. Journal of Clinical Microbiology, 52, 130-138. 
http://dx.doi.org/10.1128/JCM.01996-13

[27] Dunne Jr., W.M., Doing, K., Miller, E., Miller, E., Moreno, E., Baghli, M., Mailler, S., Girard, V., van Belkum, A. and Deol, P. (2014) Rapid Inactivation of Mycobacterium and Nocardia Species before Identification Using Matrix-Assisted Laser Desorption Ionization-Time of Flight Mass Spectrometry. Journal of Clinical Microbiology, 52, 3654-3659. http://dx.doi.org/10.1128/JCM.01728-14

[28] Balada-Llasat, J.M., Kamboj, K. and Pancholi, P. (2013) Identification of Mycobacteria from Solid and Liquid Media by Matrix-Assisted Laser Desorption Ionization-Time of Flight Mass Spectrometry in the Clinical Laboratory. Journal of Clinical Microbiology, 51, 2875-2879. http://dx.doi.org/10.1128/JCM.00819-13

[29] Telenti, A., Marchesi, F., Balz, M., Bally, F., Bottger, E.C. and Bodmer, T. (1993) Rapid Identification of Mycobacteria to the Species Level by Polymerase Chain Reaction and Restriction Enzyme Analysis. Journal of Clinical Microbiology, 31, 175-178.

[30] Selvaraju, S.B., Khan, I.U. and Yadav, J.S. (2005) A New Method for Species Identification and Differentiation of Mycobacterium chelonae Complex Based on Amplified Hsp65 Restriction Analysis (AHSPRA). Molecular and Cellular Probes, 19, 93-99. http://dx.doi.org/10.1016/j.mcp.2004.09.007

[31] Adekambi, T. and Drancourt, M. (2004) Dissection of Phylogenetic Relationships among 19 Rapidly Growing Mycobacterium Species by 16S rRNA, hsp65, sodA, recA and rpoB Gene Sequencing. International Journal of Systematic and Evolutionary Microbiology, 54, 2095-2105. http://dx.doi.org/10.1099/ijs.0.63094-0

[32] McNabb, A., Eisler, D., Adie, K., Amos, M., Rodrigues, M., Stephens, G., Black, W.A. and Isaac-Renton, J. (2004) Assessment of Partial Sequencing of the 65-Kilodalton Heat Shock Protein Gene (hsp65) for Routine Identification of Mycobacterium Species Isolated from Clinical Sources. Journal of Clinical Microbiology, 42, 3000-3011. http://dx.doi.org/10.1128/JCM.42.7.3000-3011.2004

[33] Beccati, M., Peano, A. and Gallo, M.G. (2007) Pyogranulomatous panniculitis Caused by Mycobacterium alvei in a Cat. The Journal of Small Animal Practice, 48, 664. http://dx.doi.org/10.1111/j.1748-5827.2007.00502.x

[34] Hsiao, C.H., Lin, Y.T., Lai, C.C. and Hsueh, P.R. (2010) Clinicopathologic Characteristics of Nontuberculous Mycobacterial Lung Disease in Taiwan. Diagnostic Microbiology and Infectious Disease, 68, 228-235. http://dx.doi.org/10.1016/j.diagmicrobio.2010.06.008

[35] Kim, C.K., Choi, S.I., Jeon, B.R., Lee, Y.W., Lee, Y.K. and Shin, H.B. (2014) Pulmonary Infection Caused by Mycobacterium neoaurum: The First Case in Korea. Annals of Laboratory Medicine, 34, 243-246. http://dx.doi.org/10.3343/alm.2014.34.3.243

[36] Poh, M.E., Liam, C.K., Ng, K.P. and Tan, R. (2014) Mycobacterium brisbanense Species Nova Isolated from a Patient with Chronic Cavitary Lung Infection. Chest, 145, 858-860. http://dx.doi.org/10.1378/chest.13-1952

[37] Puthalapattu, S. and Metersky, M.L. (2011) Mycobacterium nebraskense as a Cause of Nodular Pulmonary Disease. Connecticut Medicine, 75, 527-529.

[38] Lee, E.S., Lee, M.Y., Han, S.H. and Ka, J.O. (2008) Occurrence and Molecular Differentiation of Environmental Mycobacteria in Surface Waters. Journal of Microbiology and Biotechnology, 18, 1207-1215.

[39] Dai, J., Chen, Y. and Lauzardo, M. (2011) Web-Accessible Database of hsp65 Sequences from Mycobacterium Reference Strains. Journal of Clinical Microbiology, 49, 2296-2303. http://dx.doi.org/10.1128/JCM.02602-10. 